\title{
A Temporo-Spatial Regulation of Sema3c Is Essential for Interaction of Progenitor Cells during Cardiac Outflow Tract Development
}

\author{
Kazuki Kodo, Shinsuke Shibata, Sachiko Miyagawa-Tomita, \\ Sang-Ging Ong, Hiroshi Takahashi, Tsutomu Kume, \\ Hideyuki Okano, Rumiko Matsuoka, \\ and Hiroyuki Yamagishi
}

\footnotetext{
K. Kodo $\cdot$ H. Yamagishi $(\bowtie)$

Division of Pediatric Cardiology, Department of Pediatrics, Keio University School of Medicine, Tokyo, Japan

e-mail: hyamag@keio.jp

S. Shibata $\cdot$ H. Okano

Department of Physiology, Keio University School of Medicine, Tokyo, Japan

S. Miyagawa-Tomita

Department of Pediatric Cardiology, Tokyo Women's Medical University, Tokyo, Japan

Department of Physiological Chemistry and Metabolism, Graduate School of Medicine, The University of Tokyo, Bunkyo City, Tokyo, Japan

Department of Veterinary Technology, Yamazaki Gakuen University, Hachioji, Tokyo, Japan

S.-G. Ong

Department of Pharmacology, University of Illinois at Chicago,

Chicago, IL, USA

H. Takahashi

Department of Neurology, National Hospital Organization, Tottori Medical Center,

Tottori, Japan

T. Kume

Feinberg Cardiovascular Research Institute, Feinberg School of Medicine, Northwestern

University, Chicago, IL, USA

R. Matsuoka

Department of Pediatric Cardiology, TWMU, Tokyo, Japan
} 


\section{Keywords}

Cardiac neural crest cell $\cdot$ Second heart field $\cdot$ TBX1 $\cdot$ Congenital heart disease

The two cardiac progenitor cell lineages, cardiac neural crest cells (cNCCs) and the second heart field (SHF), play key roles in the development of the cardiac outflow tract (OFT). Both cardiac progenitor cells interact with each other and contribute to OFT formation cooperatively. The neurovascular guiding molecule, semaphorin 3c (Sema3c), is thought to serve as a key attractant for the migration of cNCCs. A previous study reported that Tbx1 null mice showed a significant reduction in Sema3c expression in the OFT region [1]. However, the regulatory effect of Tbx1 on Sema3c was unclear. Here, we show that Sema3c plays key roles in cNCCs-SHF interactions through the regulation by Tbx1 and other molecules during OFT development [2].

By generating reporter transgenic mice harboring Sema3c enhancer fragments and lacZ transgene, we found that Foxc1/c2 directly regulated Sema3c expression in the OFT via direct binding to the Fox-site on the 5' flanking sequence of Sema $3 c$ where Tbx1 synergistically enhanced the Foxc1/c2 function. In comparison, Tbx1 in the SHF inhibited ectopic Sema3c expression in cNCCs within the pharyngeal arch region via Fgf8-ERK signaling. Consistently, the ectopic expression of Sema3c in the cNCCs of Tbxl hypomorphic mouse embryos caused abnormal migration and/or aggregation, and the overexpression of Sema3c in cultured cNCCs inhibited their migration, resulting in their aggregation. These results suggest that Tbx1-Fgf8-ERK signaling negatively regulates Sema3c expression in cNCCs for the proper guidance of their migration from the dorsal pharyngeal region to the OFT.

In conclusion, a proper temporo-spatial expression of Sema3c, which is tightly regulated positively by Foxc1/c2 and negatively by the Tbx1-Fgf8 cascade, is essential for the correct navigation of cNCCs towards the OFT (Fig. 58.1). This report is the first evidence of a detailed signal transduction mechanism for the interaction between cNCCs and SHF progenitor cells through Sema3c, providing a new molecular basis for a potential therapeutic strategy for congenital heart diseases involving OFT defects.

This work was supported by MEXT KAKENHI, Grant-in-Aid for Scientific Research (B) and Grant-in-Aid for Young Scientists (B), Keio University Academic Development Funds and Brain/MINDS project from AMED, Japan. 


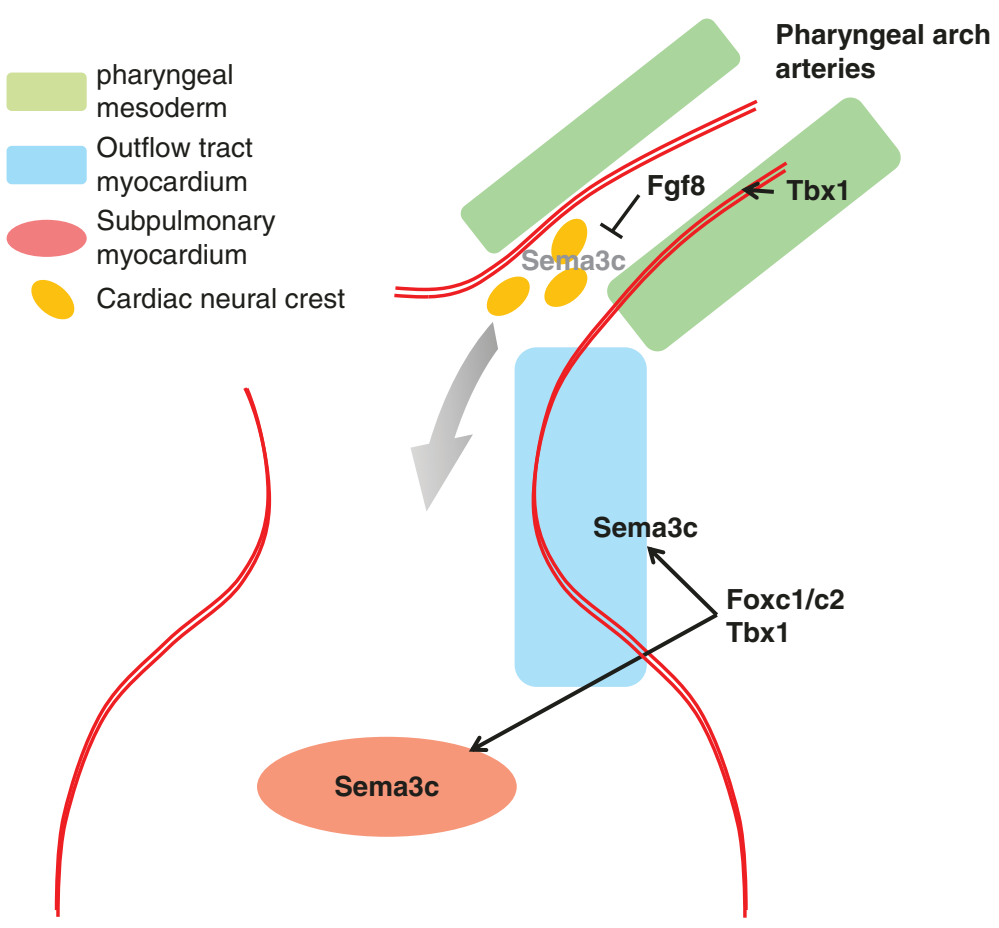

Fig. 58.1 Temporo-spatial regulation of Sema3c by Foxc1/c2 and Tbx1-Fgf8 cascade from the second heart field for proper navigation of cardiac neural crest cells towards the developing outflow tract

\section{References}

1. Kodo K, Shibata S, Miyagawa-Tomita S, et al. Regulation of Sema3c and the interaction between cardiac neural crest and second heart field during outflow tract development. Sci Rep. 2017;7:6771.

2. Théveniau-Ruissy M, Dandonneau M, Mesbah K, et al. The del22q11.2 candidate gene Tbx 1 controls regional outflow tract identity and coronary artery patterning. Circ Res. 2008;103:142-8.

Open Access This chapter is licensed under the terms of the Creative Commons Attribution 4.0 International License (http://creativecommons.org/licenses/by/4.0/), which permits use, sharing, adaptation, distribution and reproduction in any medium or format, as long as you give appropriate credit to the original author(s) and the source, provide a link to the Creative Commons license and indicate if changes were made.

The images or other third party material in this chapter are included in the chapter's Creative Commons license, unless indicated otherwise in a credit line to the material. If material is not included in the chapter's Creative Commons license and your intended use is not permitted by statutory regulation or exceeds the permitted use, you will need to obtain permission directly from the copyright holder.

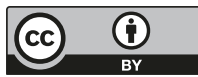

